\title{
BMJ Open What are the essential features of a successful surgical registry? a systematic review
}

\author{
Rishi Mandavia, ${ }^{1}$ Alec Knight, ${ }^{2}$ John Phillips, ${ }^{3}$ Elias Mossialos, ${ }^{4}$ Peter Littlejohns, ${ }^{2}$ \\ Anne Schilder ${ }^{1}$
}

To cite: Mandavia R, Knight A, Phillips J, et al. What are the essential features of a successful surgical registry? a systematic review. BMJ Open 2017;7:e017373. doi:10.1136/ bmjopen-2017-017373

- Prepublication history and additional material for this paper are available online. To view please visit the journal (http:// dx.doi.org/10.1136/bmjopen2017-017373).

Received 22 April 2017 Accepted 16 August 2017

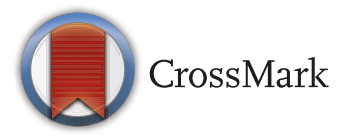

${ }^{1}$ evidENT team, UCL Ear Institute, Royal National Throat, Nose and Ear Hospital, London, UK

${ }^{2}$ Department of Primary Care and Public Health Sciences,

King's College London, London, UK

${ }^{3}$ Department of Ear Nose and Throat Surgery, Norfolk and Norwich University Hospital, Norwich, UK

${ }^{4}$ Centre for Health Policy, Imperial College London, St Mary's Hospital, London, UK

Correspondence to

Rishi Mandavia;

rishimandavia@gmail.com

\section{ABSTRACT}

Objective The regulation of surgical implants is vital to patient safety, and there is an international drive to establish registries for all implants. Hearing loss is an area of unmet need, and industry is targeting this field with a growing range of surgically implanted hearing devices. Currently, there is no comprehensive UK registry capturing data on these devices; in its absence, it is difficult to monitor safety, practices and effectiveness. A solution is developing a national registry of all auditory implants. However, developing and maintaining a registry faces considerable challenges. In this systematic review, we aimed to identify the essential features of a successful surgical registry.

Methods A systematic literature review was performed adhering to Preferred Reporting Items for Systematic Review and Meta-Analysis recommendations. A comprehensive search of the Medline and Embase databases was conducted in November 2016 using the Ovid Portal. Inclusion criteria were: publications describing the design, development, critical analysis or current status of a national surgical registry. All registry names identified in the screening process were noted and searched in the grey literature. Available national registry reports were reviewed from registry websites. Data were extracted using a data extraction table developed by thematic analysis. Extracted data were synthesised into a structured narrative.

Results Sixty-nine publications were included. The fundamentals to successful registry development include: steering committee to lead and oversee the registry; clear registry objectives; planning for initial and long-term funding; strategic national collaborations among key stakeholders; dedicated registry management team; consensus meetings to agree registry dataset; established data processing systems; anticipating challenges; and implementing strategies to increase data completion. Patient involvement and awareness of legal factors should occur throughout the development process.

Conclusions This systematic review provides robust knowledge that can be used to inform the successful development of any UK surgical registry. It also provides a methodological framework for international surgical registry development.

\section{Strengths and limitations of this study}

- This review provides a systematic and evidencebased foundation for the development of any surgical registry.

- We adopted a rigorous approach searching both the scientific and grey literature and used thematic analysis to develop our data extraction table.

- Data analyses at all stages were cross-checked by a second judge and discussed at consensus meetings.

- We did not perform quality assessment of the publications included in this review, owing to the non-empirical nature of included publications and the considerable heterogeneity among types of included publications.

- By excluding non-surgical registries, we may have failed to capture important information on registry development. Our decision was based on surgical registries having specific attributes that we wanted to learn from including: datasets, strategies to increase surgeon 'buy in', funding sources, key challenges and others.

\section{INTRODUCTION}

The effective regulation of surgical implants is vital to patient safety. The Poly Implant Prothese (PIP) breast implant and metal-onmetal hip implant scandals have identified the risks of not gathering long-term data on implants and surgical outcomes systematically. ${ }^{12}$ As such, there is a UK and European-wide drive to establish surgical registries. ${ }^{3}$ In the UK, there are a number of well-known surgical registry initiatives including: the National Joint Registry (NJR), the National Hip Fracture Database (NHFD), the National Bariatric Surgery Registry (NBSR) and others. There are currently few registry initiatives in Ear, Nose and Throat surgery, particularly within the field of hearing.

Hearing loss is an area of unmet need, ${ }^{4-7}$ and industry is targeting this field with a growing range of surgically implanted hearing devices. ${ }^{8-11}$ Currently, there is no comprehensive UK registry capturing data on 
these devices ${ }^{10}{ }^{12}$; in its absence, it is difficult to monitor safety, practices and effectiveness. ${ }^{513}$ A solution to this is developing a national registry of all auditory implants. However, developing and maintaining a surgical registry faces considerable challenges, with the majority of registries having poor rates of data completion and short life spans. ${ }^{14}{ }^{15}$ In order to develop a successful surgical registry, it is important to learn from the experiences of previous and existing registries. In this systematic review, we aimed to identify the essential features of a successful surgical registry.

\section{MATERIALS AND METHODS Registration}

This systematic review was registered on the PROSPERO database. Registration number: CRD42016039793.

\section{Design}

Systematic review and narrative synthesis.

\section{Search strategy and selection criteria}

A systematic review was performed adhering to Preferred Reporting Items for Systematic Review and Meta-Analysis (PRISMA) recommendations. ${ }^{16}$ With expert librarian support, we designed and conducted a comprehensive search of the Medline and Embase databases from inception to November 2015 using the Ovid Portal. An updated search was performed in November 2016. The search string used was ( (surgery or surgical) AND (register or registers or registry or registries) ) AND (britain $\$$ or 'united kingdom\$' or uk or england\$ or northern ireland\$ or wales $\$$ or scotland $\$$ ). The full search strategy is provided in (online supplementary appendix 1). All registry names identified in the screening process were noted and searched in the grey literature. Available national registry reports were reviewed from registry websites. We also visually scanned reference lists and searched relevant citations in the grey literature. Two authors (RM and JP) searched the literature independently and compared results at each stage of the PRISMA flow chart (figure 1). A third author (AS) arbitrated disagreements.

Criteria for publications to be included were: publications describing the design, development, critical analysis or current status of a national surgical registry. Exclusion criteria were: non-English language, publications over 10 years old and publications describing non-surgical or non UK-registries.

\section{Data extraction and synthesis}

A data extraction table was produced in Microsoft Excel, containing 20 column headings developed by the first author (RM) (see table 1). These headings were developed following immersion in the dataset and using thematic analysis to identify the key themes for data extraction. RM extracted the data, allocating relevant information from each included publication to each of the data columns described in table 1. A second author (JP) cross-checked the development of the data extraction table and the data extraction, and this process was discussed at two interim consensus meetings. Data were then synthesised by summarising the data under each column heading into a structured narrative, following the principles outlined by Popay $e t a l .{ }^{17}$

\section{RESULTS}

After duplicates were removed, titles and abstracts of 1389 publications were screened. Thirty-five additional records were identified from other sources. Fifty-nine publications fulfilled the criteria for analysis. After conducting our updated search, 10 additional publications were included, resulting in 69 publications for analysis. See figure 1 for the PRISMA flow chart.

Included publications consisted of annual registry reports and analyses, registry overview documents, editorials, commentaries, registry proposal documents and registry review articles and covered a range of surgical specialties (see box 1). (Online supplementary appendix 2) shows the full data extraction table, identifying the relevant information from each included publication.

Below is a narrative synthesis of the full data extraction table. The numerical and alphabetical digits below correspond to the data extraction columns in (online supplementary appendix 2).

\section{Registry planning}

Registry leadership and management (1.G)

Registries are typically led by steering committees comprising professional and clinical stakeholders as well as patient representatives. ${ }^{18-22}$ Steering committees should have overall responsibility for registry design, data monitoring, data analysis ${ }^{23}$ as well as strategic direction, oversight and allocation of registry resources. ${ }^{19} 212425$

It is important for registry management to receive input from both clinical and data management experts. ${ }^{2627}$ Local registry managers help maximise data completion and accuracy $^{21}$; and private companies have been employed to successfully manage several UK national registries. ${ }^{25}$ 28-30

\section{The objective(s) of a surgical registry (1.H)}

Registries should have a clear set of objectives from the outset; these often include: improving patient care, providing comparisons of standards, monitoring current practice, monitoring device durability and intervention performance, identifying variations in service provisioning as well as guiding commissioning and guideline development. ${ }^{12} 192022$ 30-32 Other aims include gaining a better understanding of disease epidemiology $1^{19233}$ and promoting future research, innovation, efficiency, transparency and patient decision making. ${ }^{2834-38}$ The addition of objectives at a later stage, after the registry is established, will likely lead to challenges. ${ }^{12} 141532$ For instance, a registry developed to improve patient care will unlikely be successful in driving research, due to the registry not being developed to collect and report on data relevant to 


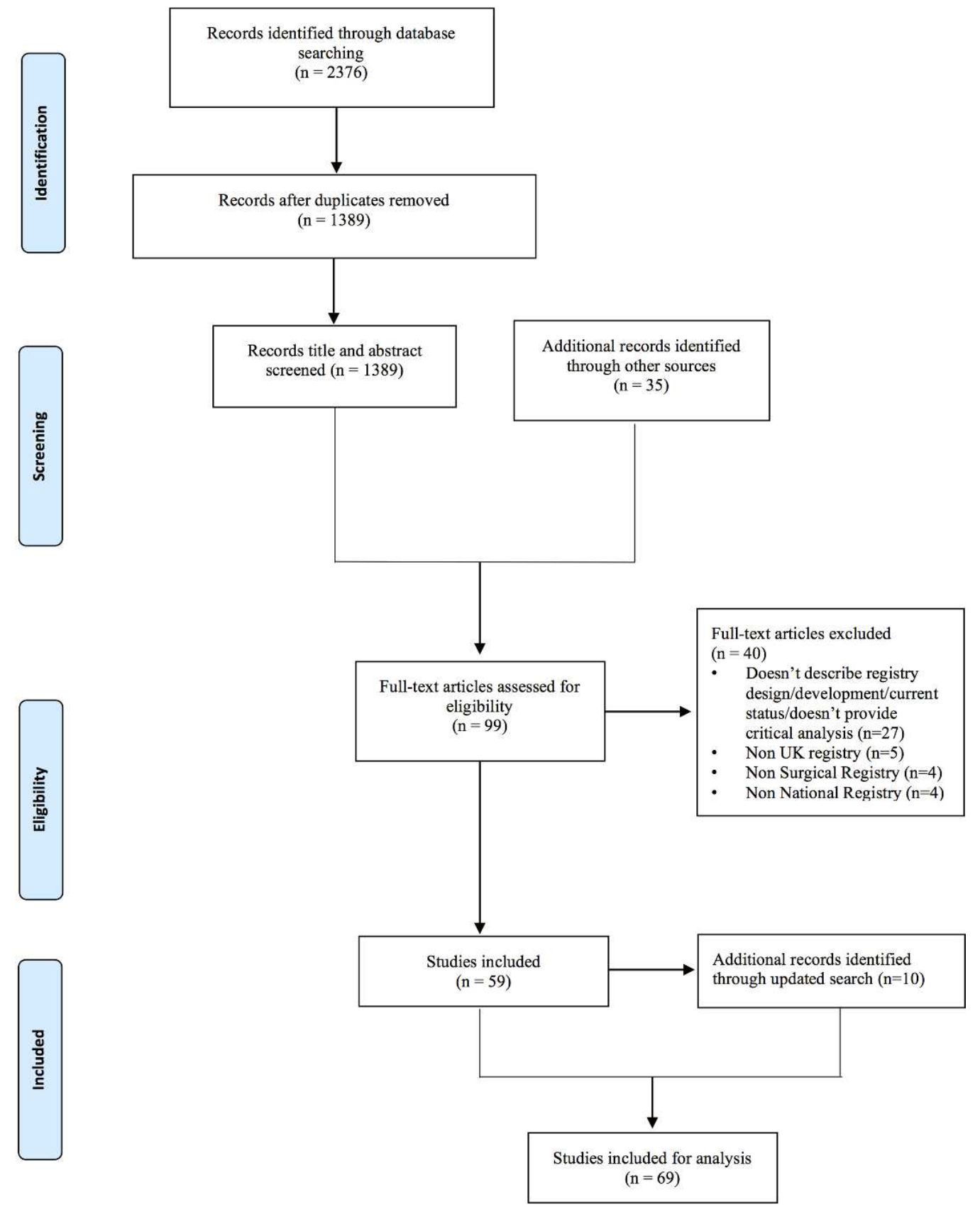

Figure 1 PRISMA diagram. PRISMA, Preferred Reporting Items for Systematic Review and Meta-Analysis.

researchers. ${ }^{12} 202223$ Registries including the NHFD, NJR and NBSR have demonstrated that by setting clear objectives from the outset and by involving key stakeholders including clinicians, patients and researchers during registry development, a registry can successfully deliver on multiple objectives, including improving patient care and driving research. ${ }^{20} 2527$

\section{Funding (1.J)}

Registries require considerable resources for initial set-up and ongoing maintenance. ${ }^{26}$ Owing to implant life span, implant registries in particular should plan for long-term funding. Central funding sources include the Healthcare Quality Improvement Partnership, National Health Service England, the Department of Health
(DOH) and national commissioners. ${ }^{22} 26{ }^{39}$ Industry can also contribute to funding, although it is important to consider governance around industry access to registry data. ${ }^{21} 294041$ Other sources of funding include participating hospitals, ${ }^{21}$ charities, ${ }^{42}$ professional societies, ${ }^{43}$ annual capitation fees ${ }^{36}$ and charging for data requests. ${ }^{26}$ Registry costs can also be incorporated into the price of each implant. ${ }^{27}$ Funding often comes from multiple sources. ${ }^{2021} 262744$

\section{Establishing collaborations (1.F)}

It is important to form strategic national collaborations among stakeholders including: patient groups, clinicians, specialist societies, industry, commissioners, funding bodies, hospitals, academic groups and those involved 
Table 1 Data column headings and their descriptions

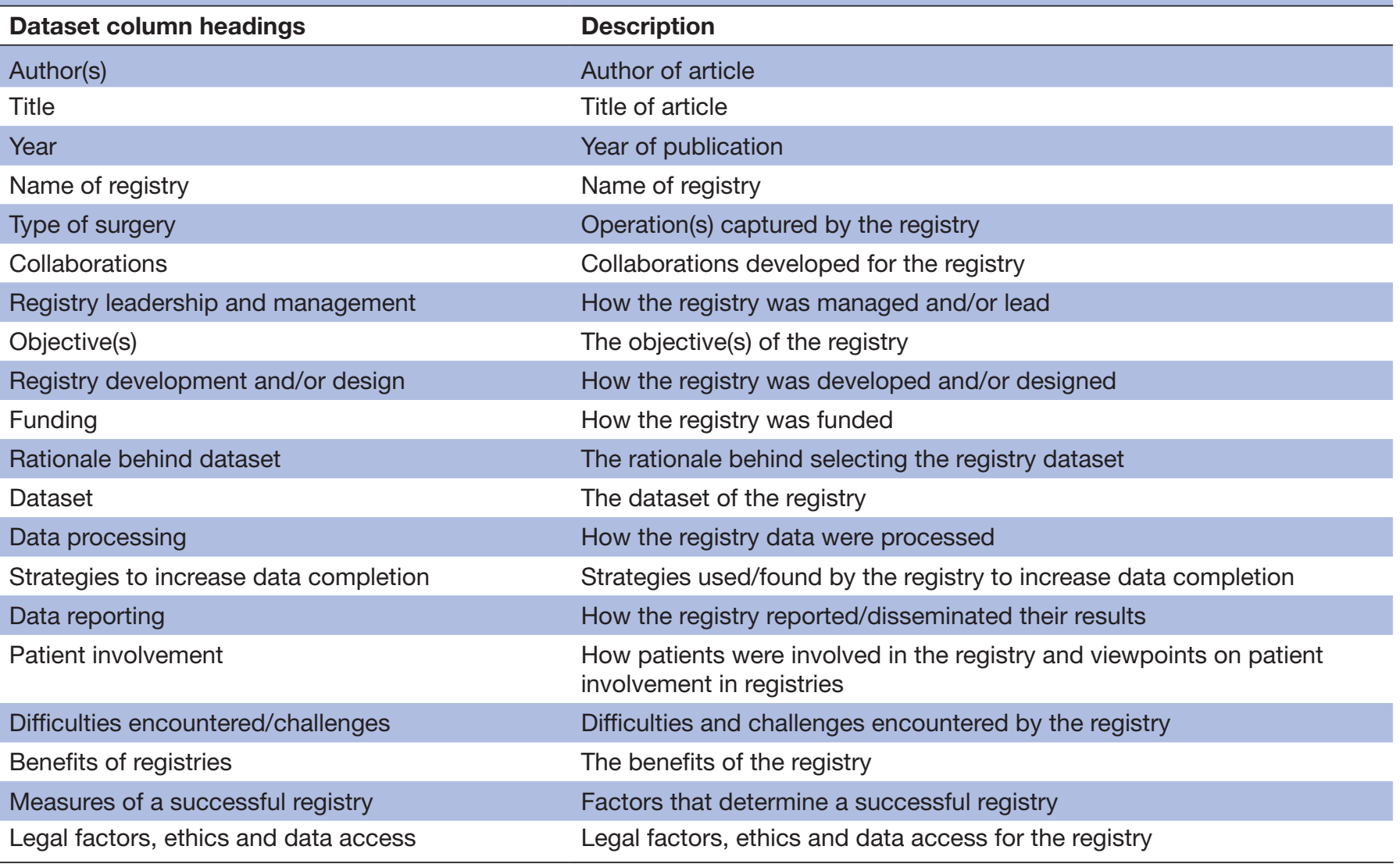

in data collection and management. ${ }^{19} 262732$ Working with and learning from existing regional registries was a successful strategy adopted by the National Vascular Registry. ${ }^{45}$ International collaborations can help align the registry with global surgical initiatives ${ }^{27} 3846$ and links with the implant industry can facilitate implant tracking. ${ }^{47}$ Collaborations with national institutes including the National Institute for Health and Care Excellence (NICE) and the Royal Colleges can align registry data with national guidelines development and revalidation. ${ }^{19}$ Collaborations with geriatrics societies and charities can help data collection on elderly patients. ${ }^{20}$

\section{Box 1 Represented surgical specialties}
- Surgical specialty
- Orthopaedics
- Renal surgery
$>$ Neurosurgery
- Cardiac surgery
- Upper gastrointestinal surgery
Urology
- Plastic surgery
- Breast surgery
- Colorectal surgery
- Cardiothoracic surgery
- Vascular surgery
- Endocrine surgery
- Ear, Nose and Throat surgery

Registry development and design (1.I)

Reaching stakeholder consensus on registry objectives, dataset and activities is essential. ${ }^{20} 3648$ The registry can be developed from existing smaller registries, ${ }^{45}$ and piloting the registry is important in obtaining user feedback. ${ }^{21} 40$ 49-51 Web-based electronic platforms facilitate quick and accurate data collection, and tailored IT systems can be developed to provide a secure, interactive and easy-to-use registry platform. ${ }^{20} 29305052$ NICE advises that registries should be recorded on a national database of registers. $^{23}$

\section{Dataset and data management}

Rationale behind a registry dataset (1.K)

It is advisable for datasets to be developed through stakeholder and patient consensus meetings, ${ }^{48} 5354$ with a balance between comprehensibility and feasibility: comprehensive datasets are unlikely to achieve data completion, while limited datasets may be less useful. ${ }^{24} 2938$ Flexible datasets built with the ability to evolve can help promote registry longevity, but an initial period of consistency helps embed the registry. ${ }^{26}{ }^{49}$ It can also be useful to build on existing registry datasets from the same specialty. $^{28} 465154$

While collecting quality of life (QoL) and patient-reported outcomes (PRO) data is vital for evaluation of treatments and services, ${ }^{55}$ collecting such data in the context of a national registry is resource intensive and may affect data completion. ${ }^{55}$ Deciding which PROs to 
Table 2 The data items collected by the majority of UK surgical registries

\begin{tabular}{|c|c|c|}
\hline Preoperative & Operative & Postoperative \\
\hline Name of centre & Name of operation & Outcome data specific to operation \\
\hline Patient identifier & Time to surgery from first appointment & QoL/PRO outcome measure \\
\hline Patient comorbidities & ASA grade & Length of stay \\
\hline Whether discussed at MDT meeting & Thromboprophylaxis regimen & Complications \\
\hline Date of diagnosis & Elective or emergency surgery & Mortality (and cause) \\
\hline Preoperative investigations and results & Date of surgery & Dates of follow-up \\
\hline Date of admission & In or out of regular hospital hours & Follow-up outcomes \\
\hline \multirow[t]{8}{*}{ GP information } & Site/side of surgery & Need for further treatment \\
\hline & Surgical technique/approach & Need for further surgery \\
\hline & Date of consent & \\
\hline & Grade of surgeon & \\
\hline & Surgical time & \\
\hline & Funding for operation (NHS/private) & \\
\hline & Use of antibiotics & \\
\hline & Type of implant and implant serial number & \\
\hline
\end{tabular}

ASA, American Society of Anaesthesiologists; GP, general practitioner; ITU, intensive therapy unit; MDT, multidisciplinary team; NHS, National Health Service; PRO patient-reported outcome; QoL, quality of life.

choose can also be an area of controversy and disagreement. ${ }^{55}$ If PROs are introduced, it is advisable to keep the number of questions short and for these data to be collected directly from patients at regular, planned time points, rather than relying on clinic follow-ups. ${ }^{305}$

The design of registry datasets can accommodate national guideline recommendations ${ }^{23} 45 \quad 57 \quad 58$; for example, the NHFD dataset is designed to facilitate easy comparison to NICE guidance, ${ }^{20}$ and the National Vascular Registry adapted datasets to capture key issues highlighted by National Confidential Enquiry into Patient Outcomes and Deaths. ${ }^{45}$

\section{Dataset (1.L)}

While specific registry data items vary between surgical specialties, the majority of UK surgical registries collect the preoperative, operative and postoperative data items summarised in table 2. A free text box can also be included to capture additional relevant information. ${ }^{30}$

\section{Data processing (1.M)}

To improve data quality and accuracy, data from participating centres should be internally validated by local registry managers and clinicians before being cleaned. $^{215960}$ Data cleaning can take place locally or centrally and involves detecting and resolving data problems. ${ }^{26} 2832$ Prior to central analysis, data can be returned to each contributing centre to take any necessary remedial actions..$^{2655961}$ On site data verification by auditors is considered good practice. ${ }^{406062}$ Although these visits focus on completeness and accuracy of data, they also provide an important opportunity for education of clinicians and local registry managers adding to ongoing data quality ${ }^{40486062}$ and for discussion with administrators about appropriate resources for information management. ${ }^{60}$ Feedback through reports evaluating quality of local data collection can be sent to contributing centres to stimulate improvements, and independent validation of data including data completeness, mortality, readmission and revision can be achieved by linking registry patient records to the Office of National Statistics and Hospital Episode Statistics (HES). ${ }^{18} 353658606263$ NICE recommends that the process for data collection, storage and analysis should be independent of any particular company or commercial interest. ${ }^{23}$

\section{Data reporting (1.P)}

Registries usually publish information via annual online comprehensive reports, ${ }^{21} 263236$ 62-64 research publications and presentations. ${ }^{27} 396265$ There is controversy surrounding the publication of surgeon-specific data. Evidence suggests that publishing these data is associated with improvements in mortality ${ }^{62}$ as well as increased transparency, patient trust and improved supervision of junior surgeons, ${ }^{2566}$ with no evidence of 'risk-adverse' surgical behaviour. ${ }^{26} 6266$ When publishing surgeon-specific outcomes, it is important to statistically adjust 
for case mix, to take into account complex, high-risk cases. ${ }^{63}{ }^{66}$ It is recommended that team level data are published to reflect that outcomes are dependent on the entire surgical team, not solely the consultant surgeon. ${ }^{66}$ Minimising the time between the surgical event and the release of data is also important for the identification of faulty implants or unsafe practices. ${ }^{63}$

\section{Challenges and data completion}

Difficulties encountered/challenges (1.R)

Registries relying on voluntary data submission are dependent on user motivation and are unlikely to achieve complete data capture. ${ }^{35} 5667$ Voluntary data submission can also result in reporting bias with under-reported complications and a non-consecutive, non-representative patient group. ${ }^{35464}$ Insufficient financial resources for registry development and maintenance is a frequent challenge ${ }^{566869}$ as is lack of stakeholder and patient 'buy-in', resulting in poor data quality and completeness. ${ }^{223143}$ Registries can be perceived to worsen documentation pressures, which may compromise data recording and limit participation. ${ }^{22}{ }^{51}$ Reaching stakeholder consensus on the registry dataset is challenging, ${ }^{22} 70$ and datasets with unclear definitions as well as those unable to adapt to changes in practice can result in difficulties in drawing national comparisons and tracking surgical activity. ${ }^{28} 31435062$ Collecting long-term follow-up data can also be challenging, particularly when patients are under the care of multiple hospitals and clinicians. ${ }^{25} 44515570$

\section{Strategies to increase data completion (1.N)}

Data completion can be optimised by careful registry design and by involving stakeholders throughout its development promoting 'buy-in'. ${ }^{25}{ }^{26}$ An online registry that is user-friendly, multibrowser compatible, simple, quick-to-use and has clear data definitions will increase data input. ${ }^{24} 26$ Other optimisation strategies include real-time data input, reminders for mandatory fields, hover-tip prompts, on-screen data validation checks, numeric limits, auto-calculations, drop-down menus, calendar support and limiting free-text fields. ${ }^{19254048505171}$ It is critical that data input is supported by allocation of dedicated time and resources, regional training sessions, succinct user guides, real-time 'chat' support, as well as email and telephone support. ${ }^{192} 4043$ Mobile 'apps' allow easy remote registry access and can also help increase data completion. ${ }^{22} 243047$

Registries that are of clear value to clinicians and institutions are more likely to achieve data completion. ${ }^{25} 263046$ For example, registry systems producing automated clinic letters or operation notes or that help record data for selfaudit and revalidation are more likely to be used. ${ }^{18} 253537$ A research friendly registry can also help increase participation, particularly if registry contributors can be listed coauthors. ${ }^{4165}$

Regular performance feedback can help maintain local interest in the registry. ${ }^{18} 1955$ The NHFD produces online graphs with live data on performance, time-to-surgery, mortality, length of stay (LOS), best practice and patient safety. ${ }^{20}$ The NJR has increased registry participation through a programme of local audits and by issuing data quality certificates that provide incentive to submit highquality data and highlight hospitals not complying with mandatory requirements. Another measure employed by the NJR is sharing cost-saving information on best implant prices, on the proviso that hospital trusts submit data to the NJR. ${ }^{27}$

Regular published reports and journal articles have been found to raise the profile of the registry, highlight non-participating units and increase data completeness and accuracy. ${ }^{60}$ Advertising can increase awareness and participation via press coverage, emails, society bulletins, letters to eligible members, conferences, regional meetings, word-of-mouth and through journal advertisements. 20354451586072

Making data input compulsory for revalidation or commissioning, or both, appears to be the most successful method of increasing data completion. ${ }^{1922} 25275160626770$

\section{Patient involvement and legal factors}

Patient involvement (1.Q)

Patient involvement in registry leadership, design, development and reporting increases the relevance of the registry to patients, commissioners and policy makers. ${ }^{18} 273136{ }^{54}$ Patients entering their own data via electronic patient portals can be particularly useful in collecting QoL and long-term follow-up data. ${ }^{22} 24304755$ To help increase registry patient participation, it is important to acquire consent early, have a registry coordinator for patient follow-up and have multiple language options. ${ }^{55}$ Facilitating patient access to data promotes transparency, patient choice and involvement. ${ }^{27} 6263$

\section{Legal factors, ethics and data access (1.U)}

UK surgical registries must comply with DOH data protection and information governance legislation for secure processing of patient healthcare data. ${ }^{21} 3653$ This process can be guided by the Data Protection Act, General Medical Council guidance, the Caldecott Confidentiality Principles and information found in the Information Governance Toolkit of the Health and Social Care Information Centre. ${ }^{36} 3973$ The registry should be implemented and reported in accordance with Declaration of Helsinki ethical principles. ${ }^{40}$ Patient informed consent should be obtained for data submission, and data should be anonymised in all cases. ${ }^{3040536070}$ Failure to function within a legal framework can result in legal termination with potential criminal repercussions. ${ }^{26}$

While easy access to the registry is essential, ${ }^{24}$ data privacy should be maintained and data should be stored securely and not shared without appropriate permissions. ${ }^{22} 2632366370$ It is important for data release to be governed under a defined data-sharing agreement, where the security and uses of the data are clearly defined. ${ }^{19} 2136$ Registries can have subcommittees or 
data managing groups that are responsible for reviewing formal access requests and ethical assessment. ${ }^{19} 293640$

\section{Registry success}

Benefits of registries (1.S)

Surgical registries can help underpin research including randomised controlled trials, assess and improve cost-effectiveness as well as inform risk prediction models. ${ }^{26} 36477475$ Other benefits include improved patient decision making, treatment development and identification of trends in practice. ${ }^{252856}$ Registries can facilitate inter(national) comparisons between centres as well as personal audit and revalidation..$^{30} 3546556775$ Publicly accessible registries can increase public trust and promote transparency and patient choice. ${ }^{61}$ With the growing number of surgical implants, registries can help identify both the highest performing and faulty implants. ${ }^{47} 7176$ The collection, feedback and publication of registry data is now a recognised way of informing clinical practice, driving quality improvement and improving patient care and safety. ${ }^{4616371}$ Since the National Audit Cardiac Surgery registry was introduced, risk-adjusted in-hospital mortality for cardiac surgery in the UK has fallen by over $50 \%$ despite more elderly and high-risk patients having surgery each year. ${ }^{26}$ Following the start of the NHFD, rates of early surgery increased from $54.5 \%$ to $71.3 \%$ and 30 -day mortality fell from $10.9 \%$ to $8.5 \% .^{20}$

Registry data can support agencies to monitor and evaluate the quality of healthcare delivered. ${ }^{20}$ They can also help identify national variations in service provisioning, map and evaluate patient pathways as well as inform health service commissioning and policy. ${ }^{37} 455658717477$ Regulatory organisations including NICE recognise the value of registries in technology assessment particularly in the absence of formal trials. ${ }^{2344} 70$ When compared with trials, registries require fewer resources and often collect data from a broader population base so their findings have strong external validity. ${ }^{41} 78$ They also frequently provide data on long-term outcomes that exceed the study window of a trial. ${ }^{65}$ They can be of particular value when investigating patient groups that are usually excluded from clinical trials such as the elderly. ${ }^{79}$

Measures of a successful registry (1.T)

A successful registry is one that is easily accessible, has a high degree of data completion and participation and helps promote inter(national) collaboration. ${ }^{22} 26636869$ They provide timely feedback to their users, identify trends in practice, improve standards of care and identify failures at the earliest opportunity. ${ }^{20} 4863$ Successful registries are useful to their stakeholders and contain validated data that are accurate and easy to analyse. ${ }^{22} 39557179$

\section{DISCUSSION}

In this systematic review, we have identified the fundamentals for developing a successful UK surgical registry. While we highlight the need for a registry of auditory implants, our findings have implications to the wider surgical community since we provide information that can be used to inform the development of any UK surgical registry.

\section{Summary of findings}

The fundamentals to successful registry development identified by this synthesis are summarised in figure 2 and include: steering committee to lead and oversee the registry; clear registry objectives; planning for initial and long-term funding; strategic national collaborations among key stakeholders; dedicated registry management team; consensus meetings to agree registry dataset; established data processing systems; anticipating challenges; and implementing strategies to increase data completion. Patient involvement and awareness of legal factors should occur throughout the developmental process.

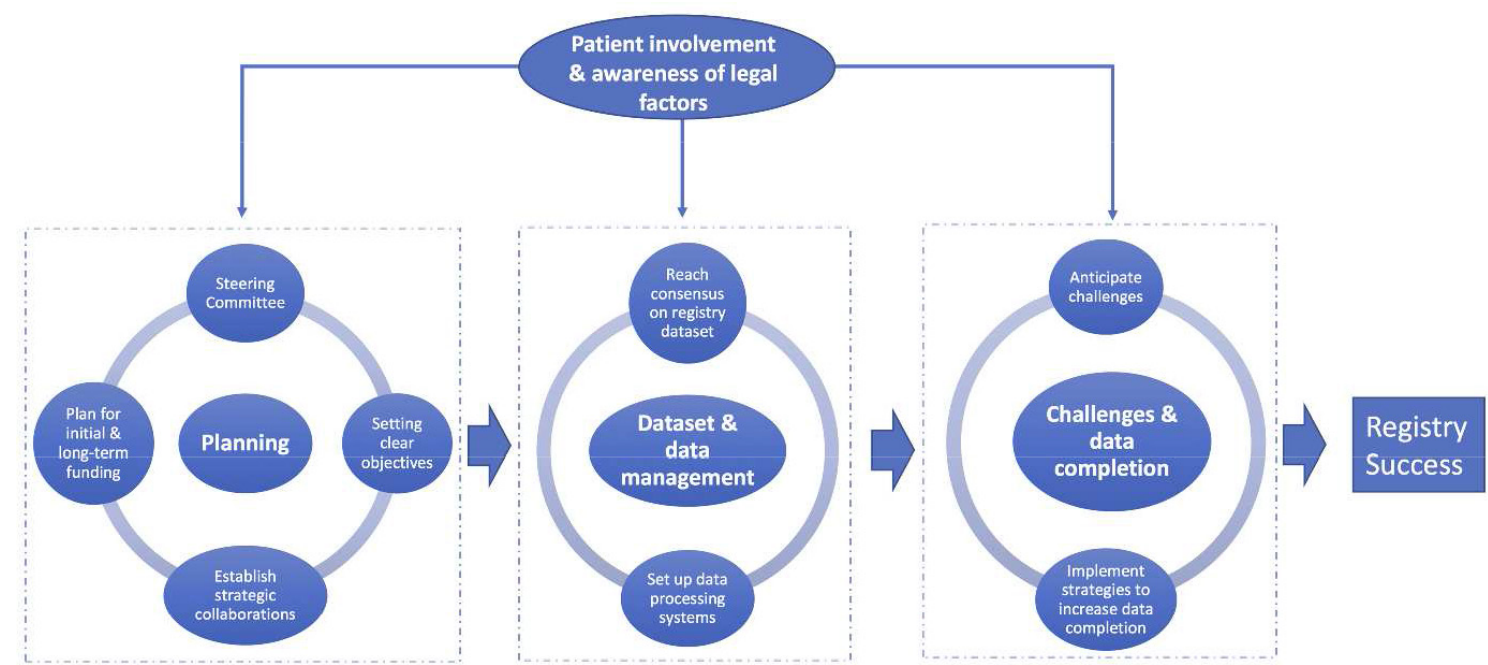

Figure 2 Overview of the key steps required for the development of a successful UK surgical registry. 


\section{Relevance to existing research}

There is a clear need for surgical registry data to improve patient safety and help regulate surgical practices. Concerns over the evidence base for surgical implants in general has been raised by the IDEAL (Idea, Development, Exploration, Assessment, Long-term monitoring) collaborative and the House of Commons Science and Technology committee. ${ }^{3} 80$ Across the UK and European Union, implants can enter surgical practice on the basis of equivalence data, meaning that an implant can be used on the basis of similarity to another implant rather than evidence of its own safety and effectiveness. ${ }^{380}$ Transparency and postmarket surveillance are additional concerns with data on safety and performance of implants not being fully published. ${ }^{3}$ The recall of the PIP breast implants and metal-on-metal hip implants identify the dangers of relying on equivalence data for the evaluation of safety and efficacy. ${ }^{12}$

Owing to these concerns, the IDEAL collaborative, DOH, NICE, policymakers and commissioning groups have called for surgical registries that can collect prospective outcome and safety data, promote transparency as well as provide patients and the public with information on their care. ${ }^{38118081}$ It has also been recognised that registry data can serve as a valuable alternative to randomised trials, which can be unfeasible and of limited scientific use, particularly at the development stage of a surgical innovation. ${ }^{4165}$ When compared with trials, registries require fewer resources, have stronger external validity and tend to provide longer term outcome data. ${ }^{4165}$

\section{Implications}

This review provides evidence-based knowledge on registry development that can be used by existing and developing UK surgical registries to increase their chance of success. Successful registries provide essential clinical and cost-effectiveness data for policy and guidelines development. $^{26477475}$ They also help develop (inter)national research collaborations as well as promote patient choice, trust and transparency. ${ }^{25} 285661$ Other implications include facilitating inter(national) benchmarking and personal audit. ${ }^{35} 46556775$ Successful registries help drive healthcare quality improvement, improve patient safety and allow commissioners and service providers to monitor quality, detect faulty implants early, monitor patient usage, identify variations in practice and allocate payments fairly. ${ }^{45} 4756717476$ From an international perspective, this review provides a methodological framework that can be adopted by other countries to promote successful national surgical registry development.

\section{Strengths and limitations}

We acknowledge that the quality and reliability of included publications likely varied due to their heterogeneity; publications included: annual registry reports and analyses, registry overview documents, editorials, commentaries, registry proposal documents and registry review articles. In addition, owing to the nature of included publications, much of the data collected were from non-empirical, opinion-based articles. This heterogeneous and non-empirical nature of included publications also precluded formal quality assessment. We recognise that the development of the data extraction table and the data extraction may have been influenced by researcher bias. However, to mitigate this, both stages were cross-checked by a second researcher and discussed at two interim consensus meetings. We also acknowledge that by excluding non-surgical registries, we may have failed to capture important information on registry development. Our decision was based on surgical registries having specific attributes that we wanted to learn from including: datasets, strategies to increase surgeon 'buy in', funding sources, key challenges and others.

A key strength of this review is that it provides an evidence-based foundation for the development of any surgical registry. We adopted a rigorous approach searching both the scientific and grey literature and used thematic analysis to develop our data extraction table. Moreover, data analyses at all stages were cross-checked by a second judge and discussed at consensus meetings.

\section{CONCLUSION}

This systematic review provides robust knowledge that can be used to inform the successful development of any UK surgical registry. It also provides a methodological framework for international surgical registry development.

Contributors RM and JP conducted the title, abstract and full-text review for this study and performed the data extraction. All authors were involved in drafting the manuscript. RM, AK, AS, EM, PL developed the search strategy. All authors were involved in conceiving the idea for this study and drafted major parts of the manuscript. All authors read and approved the final manuscript.

Funding RM was supported by a NICE Scholarship, a NIHR Academic Clinical Fellowship and a UCL Public Policy Grant. AS was supported by a NIHR Research Professorship. AK and PL were supported by the NIHR Collaboration for Leadership in Applied Health Research and Care South London at King's College Hospital NHS Foundation Trust. Funders were not involved in study design, data collection, data analysis, manuscript preparation or publication. All authors had complete access to the study data that support the publication. The views expressed are those of the authors and not necessarily those of the NHS, the NIHR or the Department of Health.

Competing interests None declared.

Provenance and peer review Not commissioned; externally peer reviewed.

Data sharing statement No additional data are available.

Open Access This is an Open Access article distributed in accordance with the Creative Commons Attribution Non Commercial (CC BY-NC 4.0) license, which permits others to distribute, remix, adapt, build upon this work non-commercially, and license their derivative works on different terms, provided the original work is properly cited and the use is non-commercial. See: http://creativecommons.org/ licenses/by-nc/4.0/

(c) Article author(s) (or their employer(s) unless otherwise stated in the text of the article) 2017. All rights reserved. No commercial use is permitted unless otherwise expressly granted.

\section{REFERENCES}

1. Oulharj S, Pauchot J, Tropet Y. PIP breast implant removal: a study of 828 cases. J Plast Reconstr Aesthet Surg 2014;67:302-7. 
2. Smith AJ, Dieppe P, Vernon K, et al. Failure rates of stemmed metalon-metal hip replacements: analysis of data from the National Joint Registry of England and Wales. Lancet 2012;379:1199-204.

3. House of Commons Science and Technology Committee. Regulation of Medical Implants in the UK and EU. 2012 http://www.publications. parliament.uk/pa/cm201213/cmselect/cmsctech/163/163.pdf (accessed 12 Oct 2016)

4. Hearing loss: an important global health concern. Lancet 2016;387:2351.

5. Committee on Accessible and Affordable Hearing Health Care for Adults, Board on Health Sciences Policy, Health and Medicine Division, National Academies of Sciences, Engineering, and Medicine. Hearing Health Care for Adults Blazer DG Domnitz S Liverman CT Washington, D C National Academies Press 2016.

6. Leverton T. Hearing loss is important in dementia. BMJ 2015;350:h3650.

7. Lamb B, ONeill C, Atkins J. The Real Cost of Adult Hearing Loss. Ear Foundation 2014. http www.earfoundation.org.uknewsarticles (accessed 5 Nov 2016).

8. Department of Health. Action Plan on Hearing Loss 2015. https:// www.england.nhs.uk/wp-content/uploads/2015/03/act-plan-hearingloss-upd.pdf.

9. NHS England 2016. Clinical Commissioning Policy: Bone conducting hearing implants (BCHIs) for hearing loss (all ages). 2016 https://www.england.nhs.uk/commissioning/wp-content/uploads/ sites/12/2013/05/16041_FINAL.pdf (accessed 15 Aug 2016).

10. NHS Commissioning Board (2013). Clinical Commissioning Policy: Bone Anchored Hearing Aids. https://www.england.nhs.uk/wpcontent/uploads/2013/04/d09-p-a.pdf (accessed 15 Aug 2016).

11. National Institute of Health \& Care Excellence. Cochlear implants for children and adults with severe to profound deafness. NICE technology appraisal guidance. 2009.

12. Ear Foundation. National Bone Conducting Hearing Implant Registry http://www.earfoundation.org.uk/hearing-technologies/boneconducting-hearing-implants/national-bone-conducting-hearingimplant-registry (accessed 14 Sep 2016).

13. GENERATE. The Research Agenda for ENT, Hearing and Balance Care.A UK Partnership of Patients, Professionals and the Public. https://entuk.org/sites/default/files/files/Research\%20Agenda\% 20ENT\%20Hearing\%20and\%20Balance\%2030\%2011\%2015.pdf (accessed 14 Sep 2016).

14. Gliklich RE, Dreyer NA, Leavy MB. Registries for Evaluating Patient Outcomes: A User's Guide. 3rd edn. Rockville (MD: Agency for Healthcare Research and Quality (US), 2014.

15. Workman TA. Engaging Patients in Information Sharing and Data Collection: The Role of Patient-Powered Registries and Research Networks. Rockville (MD: Agency for Healthcare Research and Quality (US), 2013.

16. Moher D, Altman DG, Liberati A, et al. PRISMA statement. Epidemiology 2011;22:128.

17. Popay J, Roberts H, Sowden A, et al. Guidance on the conduct of Narrative Synthesis in Systematic review. A Product from the ESRC methods programme. 2009 http://www.lancaster.ac.uk/shm/ research/nssr/research/dissemination/publications/NS_Synthesis Guidance_v1.pdf (accessed 10 Oct 2016).

18. NELA Project Board. National Emergency Laparotomy Audit (NELA) Protocol 2014

19. Ludman PF. UK TAVI Steering Group and the National Institute for Cardiovascular Outcomes Research. The UK transcatheter aortic valve implantation registry; one of the suite of registries hosted by the National Institute for Cardiovascular Outcomes Research (NICOR). Heart 2012;98:1787-9.

20. National Hip Fracture Database NHFD workstream delivery team National Hip Fracture Registry Annual Report, 2015.

21. Kolias AG, Bulters DO, Cowie CJ, et al. Proposal for establishment of the UK Cranial Reconstruction Registry (UKCRR). Br J Neurosurg 2014;28:310-4

22. Breakwell LM, Cole AA, Birch N, et al. Should we all go to the PROM? The first two years of the British Spine Registry. Bone Joint $J$ 2015;97-B-871-4.

23. National Institute for Health and Care Excellence. Interventional procedures programme manual 2016. httpswww.nice.org. ukprocesspmgchapterdraft-recommendationsdata-collection-to-a ddress-uncertainty (accessed 16 Nov 2016).

24. Gabr A, O'Leary S, Spalding T, et al. The UK National Ligament Registry Report 2015. Knee 2015;22:351-3.

25. Welbourn R, Small P, Finlay I, et al. The UK national bariatric surgery registry: The second report, 2014

26. Hickey GL, Grant SW, Cosgriff R, et al. Clinical registries: governance, management, analysis and applications. Eur J Cardiothorac Surg 2013;44:605-14.
27. The NJR Editorial Board. National Joint Registry 12th Annual Report.

28. Head SJ, Howell NJ, Osnabrugge RLJ, et al. The European Association for Cardio-Thoracic Surgery (EACTS) database: an introduction. Eur J Cardiothorac Surg 2013;44:e175-e180.

29. Chadwick D, Kinsman R, Walton P. The British Association of Endocrine and Thyroid Surgeons 4th National Audit Report, 2012.

30. Hazari A, Walton P. The UK National Flap Registry (UKNFR): A National Database for all pedicled and free flaps in the UK. J Plast Reconstr Aesthet Surg 2015;68:1633-6.

31. Briggs V, Pitcher D, Shaw C, et al. UK Renal Registry 16th annual report: chapter 142012 multisite dialysis access audit in England, Northern Ireland and Wales and 2011 PD one year followup: national and centre-specific analyses. Nephron Clin Pract 2013;125:275-94.

32. Connolly SS, Rochester MA; BAUS. Nephroureterectomy surgery in the UK in 2012: British Association of Urological Surgeons (BAUS) Registry data. BJU Int 2015;116:780-90.

33. Berven SH, Yaszemski MJ, Newton PO, et al. Introduction of new devices and technologies into a spine surgery practice: a review of processes and regulations. Orthopedics 2010;33:742-7.

34. Mason RH, Foley NM, Branley HM, et al. Pulmonary Langerhans cell histiocytosis (PLCH): a new UK register. Thorax 2014;69:766-7.

35. Armitage JN, Irving SO, Burgess NA; British Association of Urologica Surgeons Section of Endourology. Percutaneous nephrolithotomy in the United kingdom: results of a prospective data registry. Eur Urol 2012;61:1188-93.

36. Caskey F, Castledine C, Dawney A. UK Renal Registry 18th Annual Report 2016.

37. Auditbase. Advanced clinic management. 2016 https://www. auditdata.com/all-products-and-services/clinic-management/ auditbase (accessed 23 Oct 2016).

38. Yung M, Gjuric M, Haeusler R, et al. An international otology database. Otol Neurotol 2005;26:1087-92.

39. Shakespeare PG, Bazire N, Whitworth IH. The UK breast implant registry-ten years on. Br J Plast Surg 2005;58:283-5.

40. Bulusu VR, Fullarton J, Leahy M, et al. Rationale and design of a UK database for a rare cancer type: the GEM Registry for gastrointestinal stromal tumours. Br J Cancer 2013;109:1403-7.

41. Elson DW, Dawson M, Wilson C, et al. The UK Knee Osteotomy Registry (UKKOR). Knee 2015;22:1-3.

42. Bates T, Kearins O, Monypenny I, et al. Clinical outcome data for symptomatic breast cancer: the breast cancer clinical outcome measures (BCCOM) project. Br J Cancer 2009;101:395-402.

43. Uberoi R, Das N, Moss J, et al. British Society of Interventional Radiology: Biliary Drainage and Stenting Registry (BDSR). Cardiovasc Intervent Radiol 2012;35:127-38.

44. Thomas SM, Beard JD, Ireland M, et al. Results from the prospective registry of endovascular treatment of abdominal aortic aneurysms (RETA): mid term results to five years. Eur J Vasc Endovasc Surg 2005;29:563-70

45. Clinical Effectiveness Unit. National Vascular Registry 2015 Annual Report: The Royal College of Surgeons of England.

46. de Steur WO, Henneman D, Allum WH, et al. Common data items in seven European oesophagogastric cancer surgery registries: towards a European upper Gl cancer audit (EURECCA Upper Gl). Eur J Surg Oncol 2014;40:325-9.

47. Breakwell LM. Understanding the need for spinal registries: Lee Breakwell reviews the importance of registries in spinal research and explains why the British Association of Spinal Surgeons (BASS) has decided to set up its own registry. Eur Spine $J$ 2013;22:5-6.

48. Yung M, Heyning P, de HPV. A prospective multicentre otology database. Adv Otorhinolaryngol 2007;65:335-9.

49. Sher JL, Reed MR, Calvert P, et al. Influencing the national training agenda. The UK \& Ireland orthopaedic eLogbook. J Bone Joint Surg Br 2005;87:1182-6.

50. Chalmers N, Jones K, Drinkwater K, et al. The UK nephrostomy audit. Can a voluntary registry produce robust performance data? Clin Radiol 2008;63:888-94.

51. Mangera A, Parys B. BAUS Section of Endourology national Ureteroscopy audit: setting the standards for revalidation. J Clin Urol 2013;6:45-9.

52. PELICAN cancer foundation. LOREC APE Perineal Wound Registry. http://www.lorec.nhs.uk/system/content.asp?pkey=17 (accessed 20 Oct 2016).

53. Moat NE, Ludman P, de Belder MA, et al. Long-term outcomes after transcatheter aortic valve implantation in high-risk patients with severe aortic stenosis: the U.K. TAVI (United Kingdom Transcatheter Aortic Valve Implantation) Registry. J Am Coll Cardiol 2011;58:2130-8.

54. Briggs V, Pitcher D, Braddon F, et al. UK Renal Registry 15th annual report: Chapter 8 UK multisite peritoneal dialysis access 
catheter audit for first PD catheters 2011. Nephron Clin Pract 2013;123:165-81.

55. Franklin PD, Harrold L, Ayers DC. Incorporating patient-reported outcomes in total joint arthroplasty registries: challenges and opportunities. Clin Orthop Relat Res 2013;471:3482-8.

56. Bates T, Kearins O, Monypenny I, et al. Clinical outcome data for symptomatic breast cancer: the Breast Cancer Clinical Outcome Measures (BCCOM) Project. Br J Cancer 2009;101:395-402.

57. National Bowel Cancer Audit Project Team. 2015. The National Bowel Cancer Audit report.

58. Health and Social Care Information Centre. National Head and Neck Cancer Audit, 2014

59. Head SJ, Howell NJ, Osnabrugge RL, et al. The European Association for Cardio-Thoracic Surgery (EACTS) database: an introduction. Eur J Cardiothorac Surg 2013:44:e175-e180.

60. Clarke DR, Breen LS, Jacobs ML, et al. Verification of data in congenital cardiac surgery. Cardiol Young 2008;18:177-87.

61. Hickey GL, Cosgriff R, Grant SW, et al. A technical review of the United Kingdom National Adult Cardiac Surgery Governance Analysis 2008-11. Eur J Cardiothorac Surg 2014;45:225-33.

62. Bridgewater B; Society for Cardiothoracic Surgery in GB and Ireland. Cardiac registers: the adult cardiac surgery register. Heart 2010:96:1441-3.

63. Jacobs ML, Jacobs JP, Franklin RC, et al. Databases for assessing the outcomes of the treatment of patients with congenital and paediatric cardiac disease--the perspective of cardiac surgery. Cardiol Young 2008;18:101-15.

64. Wyatt MG. Registries versus trials for the evaluation of the endovascular treatment of abdominal aortic aneurysms. Eur J Vasc Endovasc Surg 2005;29:560-2.

65. Biancari F, Ruggieri VG, Perrotti A, et al. European Multicenter Study on Coronary Artery Bypass Grafting (E-CABG registry): Study Protocol for a Prospective Clinical Registry and Proposal of Classification of Postoperative Complications. J Cardiothorac Surg 2015;10:90.

66. Chou E, Abboudi H, Shamim Khan M, et al. Should surgical outcomes be published? J R Soc Med 2015;108:127-35.

67. Divecha HM, Siddique I, Breakwell LM, et al. Complications in spinal deformity surgery in the United Kingdom: 5-year results of the annual British Scoliosis Society National Audit of Morbidity and Mortality. Eur Spine J 2014;55-60.

68. Sharma S, Dreghorn CR. Registry of shoulder arthroplasty - the Scottish experience. Ann R Coll Surg Engl 2006;88:122-6.
69. Hussey K, Siddiqui T, Burton P, et al. Understanding administrative abdominal aortic aneurysm mortality data. Eur J Vasc Endovasc Surg 2015;49:277-82.

70. Hopkins J, Welbourn R. The importance of national registries/ databases in metabolic surgery: the UK experience. Surg Obes Relat Dis 2016;12:1178-85.

71. Goldberg AJ, MacGregor A, Spencer SA. An information revolution in orthopaedics. J Bone Joint Surg Br 2012;94:454-8.

72. Knight JS, Senapati A, Lamparelli MJ. National UK audit of procedure for prolapsing haemorrhoids on behalf of the Association of Coloproctology of Great Britain and Ireland. Colorectal Dis 2008;10:440-5.

73. Health and Social Care Information Centre. Information Governance Toolkit. https://www.igt.hscic.gov.uk (accessed 23 March 2016).

74. Patrick H, Sims A, Burn J, et al. Monitoring the use and outcomes of new devices and procedures: how does coding affect what Hospital Episode Statistics contribute? Lessons from 12 emerging procedures 2006-10. J Public Health 2013;35:132-8.

75. Larsson S, Lawyer P, Garellick G, et al. Use of 13 disease registries in 5 countries demonstrates the potential to use outcome data to improve health care's value. Health Aff 2012;31:220-7.

76. O'Dowd A. Government considers a national implant register in review of cosmetic procedures. BMJ 2012;345:e5574.

77. Sabah SA, Henckel J, Koutsouris S, et al. Are all metal-on-metal hip revision operations contributing to the National Joint Registry implant survival curves? : a study comparing the London Implant Retrieval Centre and National Joint Registry datasets. Bone Joint J 2016;98B:33-9.

78. Suradi HS, Hijazi ZM. TAVR update: Contemporary data from the UK TAVI and US TVT registries. Glob Cardiol Sci Pract 2015;2015:21.

79. van Gijn W, van de Velde CJ. Quality assurance through outcome registration in colorectal cancer: an ECCO initiative for Europe. Acta Chir lugos/ 2010;57:17-21.

80. McCulloch P, Cook JA, Altman DG, et al. IDEAL framework for surgical innovation 1: the idea and development stages. BMJ 2013;346:f3012.

81. NHS Commissioning Board. Clinical Commissioning Policy: Active Middle Ear Implants. https://www.england.nhs.uk/commissioning/ wp-content/uploads/sites/12/2013/04/d09-ps-a.pdf (accessed 13 Nov 2016). 